\title{
A Theoretical Approach to Study J/ $\Psi$ Suppression in Relativistic Heavy Ion Collisions with Ellipsoidal Evolution
}

\author{
Santosh K. Karn \\ Department of Physics, School of Basic Sciences and Research, Sharda University, Greater Noida, NCR-Delhi, India \\ Email: skarn03@yahoo.com, santosh.karn@sharda.ac.in
}

How to cite this paper: Karn, S.K. (2020) A Theoretical Approach to Study J/ $\Psi$ Suppression in Relativistic Heavy Ion Collisions with Ellipsoidal Evolution. Open Journal of Microphysics, 10, 1-7.

https://doi.org/10.4236/ojm.2020.101001

Received: April 1, 2019

Accepted: February 25, 2020

Published: February 28, 2020

Copyright (๑) 2020 by author(s) and Scientific Research Publishing Inc. This work is licensed under the Creative Commons Attribution International License (CC BY 4.0).

http://creativecommons.org/licenses/by/4.0/

\begin{abstract}
With a view to understanding $J / \Psi$ suppression in relativistic heavy ion collisions, we compute the suppression rate within the framework of hydrodynamical evolution model. For this, we consider an ellipsoidal flow and use an ansatz for temperature profile function which accounts for time and the three dimensional space evolution of the quark-gluon plasma. We have calculated the survival probability separately as the function of transverse and longitudinal momentum. We have shown that previous calculations are special cases of this model.
\end{abstract}

\section{Keywords}

Relativistic Heavy Ion Collisions, Quark-Gluon Plasma, Momentum Dependence, Suppression Rate, Ellipsoidal Evolution

\section{Introduction}

Relativistic heavy ion collisions vis-à-vis formation of quark-gluon plasma (QGP) and its implications have been very excited field of research [1]-[8] and are going to remain very active for a long time [2]. For a detailed study, text books and review articles on the subject, references are given in [6]. In fact, the $J / \Psi$ suppression has been theoretically argued [9] as an important signal for the formation of QGP in the collision experiments. As the science of small is deeply connected with the science of large, the study of $J / \Psi$ suppression in the evolution of QGP in such experiments has many implications in astroparticle physics and cosmological evolution as well.

In the present work, we consider ellipsoidal evolution and use exponential type temperature profile function. In the next section, we briefly outline various 
theoretical works on the understanding of $J / \Psi$ suppression. In Section 3, we compute the suppression rate within the framework of the present model. Results are discussed and summarized in Section 4.

\section{Various Theoretical Approaches}

The formation and evolution of QGP in the relativistic heavy ion collisions is well described within the framework of Bjorken hydrodynamical evolution model [10] in terms of thermodynamical variables, namely temperature ( $T$ ), entropy density $(s)$, energy density $(\varepsilon)$, etc. The probability of disintegration is related to survival probability $(S)$ which is given [11] by

$$
P=1-S=1-\exp (-T)
$$

In all the works on the $J / \Psi$ suppression, survival probability has been calculated by parameterizing the thermodynamical quantities, namely the entropy density $s$ [12], energy density $\mathcal{\varepsilon}$ [13], and temperature function $T$ [14] [15] respectively as

$$
\begin{aligned}
& s\left(t_{0}, r\right)=s_{0}\left(1-r^{2} / R^{2}\right)^{a^{\prime}} ; \varepsilon(r)=\varepsilon_{0}\left(1-r^{2} / R^{2}\right)^{2 / 3} ; \\
& T(r)=T(0)\left(1-r^{2} / R^{2}\right)^{b / 3} \text { and } \\
& T(r, t)=T_{0} \exp (-b r / R) \exp (-\eta z) t^{-1 / 3}
\end{aligned}
$$

where $R$ is the projectile radius, $a^{\prime}$ is a free parameter [12], $b$ is a parameter fixed as $1 / 3$ [14], and $\eta$ is a parameter which is a measure of steepness of the fall of temperature along z-direction [9]. In all the works except [15], the dependence of $S$ on transverse momentum $p_{T}$ has been calculated in the non transparent manner while in [15] a cylindrical interaction volume is considered and $J / \Psi$ suppression has been studied. However, in the present work, we make an attempt to consider an ellipsoidal interaction volume and compute the survival probability of $J / \Psi$ suppression which is described in the next section.

\section{Present Model}

In this section, we consider ellipsoidal evolution in both time and three dimensional space within the framework of hydrodynamical model for QGP. It is pertinent to mention here that ellipsoidal evolution has been used in physical sciences and other branches of science [16] [17] [18] [19] [20] and in other branches of science also it is being widely used [21] [22]. We feel that nature follows ellipsoidal evolution in most of the cases. Here, we use the concept of temperature density normalized to unity over the plasma volume [15] [23] [24] and compute the survival probability $S$ as a function of $p_{L}$ and $p_{T}$ respectively.

For ellipsoidal symmetry, the four dimensional temperature profile function, in general, can be written as

$$
T\left(t, \boldsymbol{r}^{\prime}\right)=T_{0} f(t, z) T(\boldsymbol{r})
$$

where $f(t, z)$ is a function of time and $z$ axis along the collision direction; $\boldsymbol{r}^{\prime}$ 
is a function of $x, y$ and $z$, and $r$ is a function of $x$ and $y$ in the transverse plane in the ellipsoidal interaction volume under consideration. Here we have considered various functions as

$$
\begin{aligned}
& f(t, z)=t^{-1 / 3} \mathrm{e}^{-\eta z} ; T(\boldsymbol{r})=\mathrm{e}^{-\alpha_{1} x} \mathrm{e}^{-\alpha_{2} y} \\
& \text { and } T(\boldsymbol{r}) f(t, z)=T\left(\boldsymbol{r}^{\prime}\right)
\end{aligned}
$$

where $\eta, \alpha_{1}$ and $\alpha_{2}$ are measure of steepness of the fall of temperature along $z, x$ and $y$ directions. Here, $\alpha_{1}=b_{1} / R_{1}, \alpha_{2}=b_{2} / R_{2}$ with $R_{1}$ and $R_{2}$ are radius of colliding nuclei and $b_{1}$ and $b_{2}$ are parameters. The time dependence of the temperature profile function given in Equation (3) is in accordance with the scaling law [10] [25]. We normalize the temperature profile function to unity over the ellipsoidal plasma volume as

$$
\int T\left(t, \boldsymbol{r}^{\prime}\right) \mathrm{d} \tau^{\prime}=1
$$

where $\mathrm{d} \tau^{\prime}$ is an ellipsoidal evolution volume element. Equations (3) and (4) lead to

$$
T_{0} \int t^{-1 / 3} \mathrm{~d} t \int \mathrm{e}^{-\eta \eta z} \mathrm{~d} z \int \mathrm{e}^{-\alpha_{1} x} \mathrm{~d} x \int \mathrm{e}^{-\alpha_{2} y} \mathrm{~d} y=1
$$

Solving integrals in Equation (6), we obtain the normalization constant as

$$
\begin{aligned}
T_{0}= & \left(\eta \alpha_{1} \alpha_{2} / 12\right)\left[\left(t_{f}^{2 / 3}-t_{i}^{2 / 3}\right) \sinh (\eta c) \cdot \sinh \left(\alpha_{1} a \sqrt{1-h^{2} / c^{2}}\right)\right. \\
& \left.\cdot \sinh \left(\alpha_{2} b \sqrt{1-h^{2} / c^{2}}\right)\right]^{-1}
\end{aligned}
$$

where $a, b$ and $c$ are along $x, y$ and $z$ directions respectively; $h$ within sine and cosine hyperbolic functions represents $\mathrm{z}$ co-ordinate such that $x$ varies from $-a \sqrt{1-h^{2} / c^{2}}$ to $+a \sqrt{1-h^{2} / c^{2}} ; y$ varies from $-b \sqrt{1-h^{2} / c^{2}}$ to $+b \sqrt{1-h^{2} / c^{2}}$ and $z$ varies from $-c$ to $+c$. The plasma evolution ellipsoidal volume, $V=\int F(h) \mathrm{d} h=(4 / 3) \pi a b c$ where the area $F(h)=\pi a b\left(1-h^{2} / c^{2}\right)$.

Therefore, the temperature profile function becomes

$$
\begin{aligned}
T\left(t, \boldsymbol{r}^{\prime}\right)= & \left(\eta \alpha_{1} \alpha_{2} / 12\right)\left[\left(t_{f}^{2 / 3}-t_{i}^{2 / 3}\right) \sinh (\eta c) \cdot \sinh \left(\alpha_{1} a \sqrt{1-h^{2} / c^{2}}\right)\right. \\
& \left.\cdot \sinh \left(\alpha_{2} b \sqrt{1-h^{2} / c^{2}}\right)\right]^{-1} \cdot t^{-1 / 3} \mathrm{e}^{-\eta z} \mathrm{e}^{-\left(\alpha_{1} x+\alpha_{2} y\right)}
\end{aligned}
$$

As momentum $\boldsymbol{p}$ is the conjugate of position vector $\boldsymbol{r}^{\prime}$, we take the Fourier transforms of $T\left(t, \boldsymbol{r}^{\prime}\right)$ in Equation (3) with respect to $\boldsymbol{r}^{\prime}$ which finally leads to $p_{L}$ and $p_{T}$ dependences of survival probability $S$. Therefore, we write

$$
T\left(p_{L}, p_{T}\right)=\int T\left(t, \boldsymbol{r}^{\prime}\right) \mathrm{e}^{i p^{\prime} \cdot \boldsymbol{r}^{\prime}} \mathrm{d} t \mathrm{~d} \tau^{\prime}
$$

From Equation (9), we obtain

$$
\left|T\left(p_{L}, p_{T}\right)\right|=(3 / 2) T_{0}\left(t_{f}^{2 / 3}-t_{i}^{2 / 3}\right)\left|I_{z}\right|\left|I_{x} \|\right| I_{y} \mid
$$

where $\left|I_{z}\right|=\sqrt{I_{z} I_{z}^{*}}, \quad\left|I_{x}\right|=\sqrt{I_{x} I_{x}^{*}}, \quad\left|I_{y}\right|=\sqrt{I_{y} I_{y}^{*}} \quad$ with $\quad I_{z}=\int \mathrm{e}^{i p_{L} z} \mathrm{e}^{-\eta \eta z} \mathrm{~d} z$, $I_{x}=\int \mathrm{e}^{i i_{T X} x} \mathrm{e}^{-\alpha_{1} x} \mathrm{~d} x$, and $I_{y}=\int \mathrm{e}^{i p_{T y} y} \mathrm{e}^{-\alpha_{2} y} \mathrm{~d} y$. 
Therefore, we obtain

$$
\begin{aligned}
\left|T\left(p_{L}, p_{T}\right)\right|= & \frac{\eta\left[\sinh ^{2} \eta c \cos ^{2} p_{L} c+\cosh ^{2} \eta c \sin ^{2} p_{L} c\right]^{1 / 2}}{\sinh \eta c \cdot\left(\eta^{2}+p_{L}^{2}\right)^{1 / 2}} \\
& \cdot \alpha_{1} \frac{\left[\sinh ^{2} \alpha_{1} a\left(1-z^{2} / c^{2}\right)^{\frac{1}{2}} \cos ^{2} p_{T x} a\left(1-z^{2} / c^{2}\right)^{\frac{1}{2}}+\cosh ^{2} \alpha_{1} a\left(1-z^{2} / c^{2}\right)^{\frac{1}{2}} \sin ^{2} p_{T x} a\left(1-z^{2} / c^{2}\right)^{\frac{1}{2}}\right]^{1 / 2}}{\left(\alpha_{1}^{2}+p_{T x}^{2}\right)^{1 / 2} \sinh \alpha_{1} a\left(1-z^{2} / c^{2}\right)^{\frac{1}{2}}} \\
& \cdot \alpha_{2} \frac{\left[\sinh ^{2} \alpha_{2} b\left(1-z^{2} / c^{2}\right)^{\frac{1}{2}} \cos ^{2} p_{T y} b\left(1-z^{2} / c^{2}\right)^{\frac{1}{2}}+\cosh ^{2} \alpha_{2} b\left(1-z^{2} / c^{2}\right)^{\frac{1}{2}} \sin ^{2} p_{T y} b\left(1-z^{2} / c^{2}\right)^{\frac{1}{2}}\right]^{1 / 2}}{\left(\alpha_{2}^{2}+p_{T y}^{2}\right)^{1 / 2} \sinh \alpha_{2} b\left(1-z^{2} / c^{2}\right)^{\frac{1}{2}}}
\end{aligned}
$$

From Equation (11), we obtain the value of $\left|T\left(P_{T}\right)\right|$ and $\left|T\left(P_{L}\right)\right|$. When $p_{L} \rightarrow 0$, we obtain an expression for $\left|T\left(P_{T}\right)\right|$ and the expression for $\left|T\left(P_{L}\right)\right|$ is obtained by taking $p_{T} \rightarrow 0$. The obtained expressions [26] are

$$
\begin{aligned}
\left|T\left(p_{T}\right)\right|= & \alpha_{1} \frac{\left[\sinh ^{2} \alpha_{1} a\left(1-z^{2} / c^{2}\right)^{\frac{1}{2}} \cos ^{2} p_{T x} a\left(1-z^{2} / c^{2}\right)^{\frac{1}{2}}+\cosh ^{2} \alpha_{1} a\left(1-z^{2} / c^{2}\right)^{\frac{1}{2}} \sin ^{2} p_{T x} a\left(1-z^{2} / c^{2}\right)^{\frac{1}{2}}\right]^{1 / 2}}{\sinh \left(\alpha_{1} a\left(1-z^{2} / c^{2}\right)^{\frac{1}{2}}\right) \cdot\left(\alpha_{1}^{2}+p_{T x}^{2}\right)^{1 / 2}} \\
& \alpha_{2} \frac{\left[\sinh ^{2} \alpha_{2} b\left(1-z^{2} / c^{2}\right)^{\frac{1}{2}} \cos ^{2} p_{T y} b\left(1-z^{2} / c^{2}\right)^{\frac{1}{2}}+\cosh ^{2} \alpha_{2} b\left(1-z^{2} / c^{2}\right)^{\frac{1}{2}} \sin ^{2} p_{T y} b\left(1-z^{2} / c^{2}\right)^{\frac{1}{2}}\right]^{1 / 2}}{\sinh \alpha_{2} b\left(1-z^{2} / c^{2}\right)^{\frac{1}{2}} \cdot\left(\alpha_{2}^{2}+p_{T y}^{2}\right)^{\frac{1}{2}}}
\end{aligned}
$$

and

$$
\left|T\left(p_{L}\right)\right|=\frac{\eta}{\sinh \eta c \cdot\left(\eta^{2}+p_{L}^{2}\right)^{1 / 2}}\left[\sin ^{2} h \eta c \cos ^{2} p_{L} c+\cos ^{2} h \eta c \sin ^{2} p_{L} c\right]^{1 / 2}
$$

The expression for survival probability $S$ of the J/ $\Psi$ suppression in the relativistic heavy ion collisions is obtained by substituting the value of $\left|T\left(P_{T}\right)\right|$ and $\left|T\left(P_{L}\right)\right|$ from Equations (12) and (13) in Equation (1). It is to be noted here that Equation (5) ensures the dimensionless character of $\left|T\left(P_{T}\right)\right|$ and $\left|T\left(P_{L}\right)\right|$ given in Equations (12) and (13) and subsequently that of $S$ in Equation (1). It is seen that the expression for suppression rate of momentum along longitudinal direction in the ellipsoidal evolution remains the same as that in cylindrical evolution, i.e. Equation (13) is the same as Equation (6) in [9]. But the expression for suppression rate of momentum along transverse direction in the ellipsoidal evolution given by Equation (12) does not remain the same as obtained by others [6] [7] [8] [9]. That is transverse momentum evolution is different in ellipsoidal flow than in other types of evolution considered, namely the spherical or, the cylindrical evolutions. However, the expression for suppression rate of momentum along transverse direction in the cylindrical and spherical evolutions can be reproduced simply by substituting $a=b$ and $a=b=c$ respectively in Equation (12). That is, the previous calculations are special cases of the present 
work. In order to understand the data of the $J / \Psi$ suppression completely we argue that it is necessary to understand together the suppression rate of momentum along the longitudinal direction and the transverse direction. The total energy can be obtained by using Equation (3). In fact, by substituting the value of $T(t, \boldsymbol{r})$ from Equation (3) in the energy density equation $\varepsilon(t, r)=\left(\alpha_{S} \pi^{2} / 15\right) T^{4}\left(t, r^{\prime}\right)$, one can obtain the expression for suppression rate of energy density along the longitudinal and transverse directions. The total energy can also be obtained from the equation, $E=(4 / 3) \pi a b c \cdot \varepsilon$.

\section{Discussion and Summary}

Within the framework of the Bjorken hydrodynamical model we have tried to understand the time and space evolution of QGP by considering ellipsoidal flow of the fluid and exponential fall of temperature in longitudinal and transverse directions. In the present work we have calculated the J/ $\Psi$ suppression in the relativistic heavy ion collisions not only of transverse momentum $P_{T}$ but also of longitudinal momentum $P_{L}$. Presently available data [27]-[32] are not sufficient for longitudinal momentum for comparison with our predicted results. Therefore further experimental works for extracting the information on this aspect are desirable. In fact, the study of the survival probability with respect to $P_{T}$ and $P_{L}$ dependences together will throw light for the complete understanding of the phenomenon of $J / \Psi$ suppression. It is to note here that from the results thus obtained, one can also calculate the dependence of $J / \Psi$ suppression with transverse and longitudinal energy density by using the relation between energy density and the corresponding temperature, $\varepsilon(t, r)=\left(\alpha_{s} \pi^{2} / 15\right) T^{4}\left(t, r^{\prime}\right)$, and the results can be compared with the experimental data which we hope to address in the upcoming research works.

\section{Acknowledgements}

The author is greatly benefited from discussion with Professor H. Satz at T I F R and he wishes to express his gratitude to him. Author also wishes to thank Prof. R. S. Kaushal and Prof. Permanand for discussions. The author also wishes to thank the management of PCCS, Dr. APJ Abdul Kalam Technical University, India where the author worked for quite a long time, i.e. from November 24, 1999 to October 03, 2018 forenoon. Thanks are also due to the Head of the Department of Physics, Dean of the School of Basic Sciences and Research, and Dean, RTDC, Sharda University for providing the facilities to complete the work. The author also wishes to thank Prof. Ashok Kumar and Suvrat Karn for helping me in the typing work.

\section{Conflicts of Interest}

The author declares no conflicts of interest regarding the publication of this paper. 


\section{References}

[1] Collins, J.C. and Perry M.J. (1975) Superdense Matter: Neutrons or Asymptotically Free Quarks?. Phys. Rev. Lett., 34, 1353. https://doi.org/10.1103/PhysRevLett.34.1353

[2] Baym, G. and Chin, S.A. (1976) Can a Neutron Star Be a Giant MIT Bag? Phys Lett. B, 62, 241. https://doi.org/10.1016/0370-2693(76)90517-7

[3] Van Hove, L. (1982) Multiplicity Dependence of $\mathrm{p}(\mathrm{T})$ Spectrum as a Possible Signal for a Phase Transition in Hadronic Collisions. Phys. Lett. B, 118, CERN Preprint No. TH-5069/88.

[4] McLerran, L. (1986) The Physics of the Quark-Gluon Plasma. Rev. Mod. Phys., 58, 1021. https://doi.org/10.1103/RevModPhys.58.1021

[5] Cleymans, J., Gavai, R.V. and Suhonen, E. (1986) Quarks and Gluons at High Temperatures and Densities. Phys. Rep., 130, 217. https://doi.org/10.1016/0370-1573(86)90169-9

[6] Bhalerao, R.S. (2014) Relativistic Heavy-Ion Collisions arXiv:1404.3294v1 [nucl-th] CERN-2014-001 and KEK-Proceedings-2013-8, 219-239.

[7] Satz, H. (2004) The SPS Heavy Ion Programme. arXiv: hep-ph/ 0405051v3

[8] Satz, H. (2011) The Quark-Gluon Plasma A Short Introduction. arXiv: 1101.3937 v1 (hep-ph)

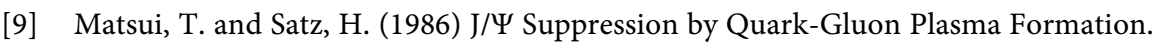
Phys. Lett. B, 178, 416. https://doi.org/10.1016/0370-2693(86)91404-8

[10] Bjorken, J.D. (1983) Highlyrelativistic Nucleus-Nucleus Collisions: The Central Rapidity Region. Phys. Rev.D, 27, 140. https://doi.org/10.1103/PhysRevD.27.140

[11] Ftacnik, J., Lichard, P., Pisutova, N. and Pisut, J. (1989) Dependence of J/I Suppression in Heavy Ion Collisions on Total Transverse Energy, Nucleon Numbers and $\mathrm{J} / \Psi$ Transverse Momentum. Z. Phys. C, 42, 139. https://doi.org/10.1007/BF01565136

[12] Blaizot, J.P. and Ollitrault, J.Y. (1987) J/ M Momentum Distribution and Lifetime of a Quark-Gluon Plasma. Phys. Lett. B, 199, 499. https://doi.org/10.1016/0370-2693(87)91616-9

[13] Karsch, F. and Satz, H. (1991) The Spectral Analysis of Strongly Interacting Matter. Z. Phys. C, 51, 209. https://doi.org/10.1007/BF01475790

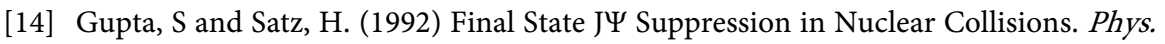
Lett. B, 383, 439. https://doi.org/10.1016/0370-2693(92)90045-6

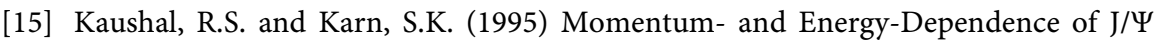
Suppression in Relativistic Heavy Ion Collisions. Pramana-Journal of Phys., 44, 2167. https://doi.org/10.1007/BF02847696

[16] Chandrasekhar, S. (1967) Ellipsoidal Figures of Equilibrium: An Historical Account. Communications on Pure and Applied Mathematics, XX, 251-265.

[17] (1969) Yale University Press, New Haven, Ch.I-VIII.

[18] Dover, Reprint, (1987) Box-95, 96, 97, 108. https://www.lib.uchicago.edu

[19] Singh, V. (2011) S Chandrasekhar. Indian Academy of Sciences, 0960-0986.

[20] Detweiler, S. and Lindblom, L. (1977) On the Evolution of the Homogeneous Ellipsoidal Figures. The Astrophysical Journal, 213, 193-199.

[21] Tan, W.-F., et al. (2013) Shape Evolution Synthesis of Monodisperse Spherical, Ellipsoidal, and Elongated Hematite $\left(\alpha-\mathrm{Fe}_{2} \mathrm{O}_{3}\right)$ Nanparticles Using Ascorbic Acid. 
Crystal Growth and Design, 14, 157-164.

[22] Seral, O. and Cuneyt, Y. (2015) On the Evolution of Ellipsoidal Recognition Regions in Artificial Immune Systems. Applied Soft Computing, 31, 210-222.

[23] Karn, S.K. and Kaushal, R.S. (1992) Transverse Momentum Dependence of the J/ $\Psi$ Suppression in Relativistic Heavy Ion Collisions (RHIC). Proc. DAE Nucl. Phys. Symp. Bombay B, 35, 378.

[24] Cooper, F., Frye, G. and Schonberg, E. (1975) Landau's Hydrodynamic Model of Particle Production and Electro-Positron Annihilation into Hadrons. Phys. Rev. D, 11, 192. https://doi.org/10.1103/PhysRevD.11.192

[25] Baym, G., Friman, B.L., Blaizot, J.P., Soyeur, M. and Czyz, W. (1983) Hydrodynamics of Ultra-Relativistic Heavy Ion Collisions. Nucl. Phys. A, 407, 541. https://doi.org/10.1016/0375-9474(83)90666-8

[26] Karn, S.K. (2020) A Theoretical Approach to Study J/ $\Psi$ Suppression in Relativistic Heavy Ion Collisions. arXiv: 2020.02412v1[nucl-th][hep-ph]SKKarn-arXiv, 2020-ui.adsabs.harvard.edu

[27] Adare, A., et al. (2010) Enhanced Production of Direct Photons in Au + Au Collisions at $\sqrt{\mathrm{S}_{\mathrm{NN}}}=200 \mathrm{GeV}$ and Implications for the Initial Temperature. Phys. Rev. Lett., 104, 132301.

[28] Abelve, B., et al. (2013) Centrality, Rapidity and Transverse Momentum Dependence of $\mathrm{J} / \Psi$ Suppression in $\mathrm{Pb}+\mathrm{Pb}$ Collisions at $\sqrt{\mathrm{S}_{\mathrm{NN}}}=2.76 \mathrm{TeV}$. [ALICE Collab.] arXiv:1311.0214[nucl.-ex] Phys. Rev. Lett., 110, 082302.

[29] Abbas, E., et al. (2013) Centrality Dependence of the Pseudorapidity Density Distribution for Charged Particles in $\mathrm{Pb}+\mathrm{Pb}$ Collisions at $\sqrt{\mathrm{S}_{\mathrm{NN}}}=2.76 \mathrm{TeV}$. [ALICE Collab.], Phys. Lett. B, 726, 610. https://doi.org/10.1016/j.physletb.2013.09.022

[30] Adler, S.S., et al. (2007) High Transverse Momentum $\eta$ Meson Production in $p+p$, $\mathrm{d}+\mathrm{Au}$, and $\mathrm{Au}+\mathrm{Au}$ Collisions at $\sqrt{\mathrm{S}_{\mathrm{NN}}}=200 \mathrm{GeV}$. [PHENIX Collab.], Phys. Rev. $C, 75,0249049$

[31] Reygers, K., et al. (2012) A Quick Tour of Ultra-Relativistic Heavy-Ion Physics at the LHC. [ALICE Collab.], arXiv:1208.1626 [nucl.-ex.]

[32] Stachel, J., et al. (2013) Confronting LHC Data with the Statistical Hadronization model. arXiv : 1311.4662 [nucl-th] 